\title{
Genomic predictions for New Zealand dairy bulls and integration with national genetic evaluation
}

\author{
B. L. Harris and D. L. Johnson ${ }^{1}$ \\ Livestock Improvement Corp., Private Bag 3016, Hamilton 3240, New Zealand
}

\section{ABSTRACT}

A method is described for the prediction of breeding values incorporating genomic information. The first stage involves the prediction of genomic breeding values for genotyped individuals. A novel component of this is the estimation of the genomic relationship matrix in the context of a multi-breed population. Because not all ancestors of genotyped animals are genotyped, a selection index procedure is used to blend genomic predictions with traditional ancestral information that is lost between the process of deregression of the national breeding values and subsequent re-estimation using the genomic relationship matrix. Finally, the genomically enhanced predictions are filtered through to nongenotyped descendants using a regression procedure.

Key words: genomic selection, relationship matrix, mixed model, reliability

\section{INTRODUCTION}

Traditional breeding value (BV) estimation combines performance and pedigree information in which degree of identity by descent is based on probabilities derived from relationships among animals in the known pedigree. An additional source of information based on DNA data can be included to provide genomic breeding values. The DNA information facilitates tracing the inheritance of individual small chromosomal segments and thereby provides more precise identity by descent information than can be derived from pedigree information.

Genetic similarity based on thousands of markers spread across the genome can be measured more accurately than similarity based on pedigree relationships (Meuwissen, 2007). Single nucleotide polymorphism markers now cover the bovine genome with high density. Genomic predictions can be based on a BLUP-GS model in which the average relationship matrix based on pedigree in the traditional BLUP model is replaced

Received August 5, 2009.

Accepted November 10, 2009.

${ }^{1}$ Corresponding author: djohnson@lic.co.nz by a genomic relationship matrix based on markers (Habier et al., 2007).

The DNA information leads to an increase in the accuracy of prediction of breeding values and a decrease in generation intervals, which facilitates selection at a young age, thus reducing the cost of progeny testing (Meuwissen et al., 2001; Schaeffer, 2006).

The objectives of this research were to apply genomic prediction methods to a population of Holstein-Friesian, Jersey, and Friesian $\times$ Jersey crossbred bulls and to integrate this information into the New Zealand genetic evaluation system for dairy cattle. The realized predictive ability of the genomic evaluations for bulls is also reported.

\section{MATERIALS AND METHODS}

\section{SNP Data}

The Illumina BovineSNP50 BeadChip (Illumina, San Diego, CA), developed by Van Tassell et al. (2008), provides genome-wide dense-marker data. Currently, Livestock Improvement Corp. (LIC; Hamilton, New Zealand) has genotyped 5,212 animals born since 1980 using this SNP panel: 2,711 Holstein-Friesian (HF), 1,738 Jersey (JE), and 763 Friesian-Jersey crossbred (FJ) bulls. After removing SNP for low call rates, minor allele frequencies $<2 \%$, non-Mendelian inheritance, and failed Hardy-Weinberg tests, a total of 44,146 SNP were retained. A further $1,844 \mathrm{SNP}$ that showed nearperfect collinearity with another $\mathrm{SNP}\left(\mathrm{R}^{2}>0.975\right)$ were removed.

\section{Linear Model and Genomic Relationships}

Let

$$
\mathbf{y}=\mathbf{X b}+\mathbf{Z u}+\mathbf{e}
$$

be the linear model relating phenotype $\mathbf{y}$ to SNP marker effects $\mathbf{u}$, where $\mathbf{X b}$ represents fixed effects and $\mathbf{e}$ is random error with diagonal variance matrix $\mathbf{R}$. The matrix $\mathbf{Z}$ has the $m$ th column vector corresponding to SNP marker $m$ and is coded $-1,0$, and 1 
for homozygote, heterozygote, and other homozygote, respectively.

The sum over all SNP loci is assumed to equal the vector of breeding values, $\mathbf{a}=\mathbf{Z} \mathbf{u}$. The $\mathrm{BV}$ can be estimated from (assume fixed effects known)

$$
\hat{\mathbf{a}}=\mathbf{Z} \hat{\mathbf{u}}=\mathbf{Z}\left(\mathbf{Z}^{\prime} \mathbf{R}^{-1} \mathbf{Z}+\mathbf{D}_{u}{ }^{-1}\right)^{-1} \mathbf{Z}^{\prime} \mathbf{R}^{-1}(\mathbf{y}-\mathbf{X b}),
$$

where $\mathbf{D}_{u}=\operatorname{var}(\mathbf{u})$ is a diagonal matrix of SNP variances. Matrix manipulation yields

$$
\hat{\mathbf{a}}=\mathbf{Z} \mathbf{D}_{u} \mathbf{Z}^{\prime}\left(\mathbf{Z} \mathbf{D}_{u} \mathbf{Z}^{\prime}+\mathbf{R}\right)^{-1}(\mathbf{y}-\mathbf{X} \mathbf{b}),
$$

and this can be further rearranged as

$$
\hat{\mathbf{a}}=\left(\mathbf{R}^{-1}+\sigma_{u}^{-2}\left(\mathbf{Z Z}^{\prime}\right)^{-1}\right)^{-1} \mathbf{R}^{-1}(\mathbf{y}-\mathbf{X b}),
$$

where it is assumed $\mathbf{D}_{u}=\sigma_{u}^{2} \mathbf{I}$; that is, all SNP variances are equal in this BLUP approach.

Similar to Habier et al. (2007), $\mathbf{Z Z}^{\prime}$ has expectation

$$
E\left(\mathbf{Z Z}^{\prime}\right)=\mathbf{A} \sum_{m} 2 p_{m} q_{m}+\mathbf{1 1 ^ { \prime }} \sum_{\mathbf{m}}\left(p_{m}-q_{m}\right)^{2}
$$

where $p_{m}$ is allele frequency $q_{m}=1-p_{m}$ and $\mathbf{A}$ is the average relationship matrix. Diagonals of $\mathbf{Z Z}^{\prime}$ count the number of homozygous loci for each individual, and off-diagonals (added to the number of SNP) measure the number of alleles shared by 2 individuals. Based on the above expectation, a genomic relationship matrix can be estimated by a regression technique (VanRaden, 2008) using the model

$$
\mathbf{Z Z}^{\prime}=b_{1} \mathbf{1 1}^{\prime}+b_{2} \mathbf{A}+\mathbf{E}
$$

where $\mathbf{E}$ includes differences between true and expected fractions of DNA in common as well as measurement error caused by using a subset of the full DNA sequence. This regression method does not require estimates of allele frequencies (which should reflect founder population values) and, as shown by VanRaden (2008), was at least as good as, if not better than, 2 other methods that required estimates of allele frequencies. The expectations of the regression coefficients are

$$
\begin{aligned}
& E\left(b_{1}\right)=\sum_{m}\left(p_{m}-q_{m}\right)^{2} \\
& E\left(b_{2}\right)=\sum_{m} 2 p_{m} q_{m} .
\end{aligned}
$$

In [6], $\mathbf{A}$ is the expected value of the genomic relationship matrix, G, and (analogous to a calibration model) $\mathbf{G}$ can be estimated by

$$
\mathbf{G}=\frac{\mathbf{Z Z}^{\prime}-\hat{b}_{1} \mathbf{1 1 ^ { \prime }}}{\hat{b}_{2}} .
$$

Substituting $\mathbf{Z Z} \mathbf{Z}^{\prime}$ from [8] into [4] and replacing regression coefficients by expected values from [7] yields, similar to VanRaden (2008),

$$
\hat{\mathbf{a}}=\left(\mathbf{R}^{-1}+\sigma_{a}^{-2} \mathbf{G}^{-1}\right)^{-1} \mathbf{R}^{-1}(\mathbf{y}-\mathbf{X b}),
$$

where

$$
\sigma_{a}^{2}=2 \sum_{m} p_{m} q_{m} \sigma_{u}^{2}
$$

is additive genetic variance. Note that the intercept term (the term involving $b_{1}$ ) disappears because solutions to the mixed-model equations are invariant to the addition of a constant to the relationship matrix. A solution strategy that avoids inverting $\mathbf{G}$ is

$$
\hat{\mathbf{a}}=\mathbf{G}\left(\mathbf{G}+\sigma_{a}^{-2} \mathbf{R}\right)^{-1}(\mathbf{y}-\mathbf{X b}),
$$

with reliabilities obtained for individual $i$ as

$$
\frac{\left[\mathbf{G}\left(\mathbf{G}+\sigma_{\mathrm{a}}^{-2} \mathbf{R}\right)^{-1} \mathbf{G}\right]_{\mathrm{ii}}}{\mathbf{G}_{\mathrm{ii}}}
$$

Expressions [11] and [12] are similar to results in VanRaden (2008) except that [12] takes account of inbreeding.

\section{Genomic Relationship Matrix for a Multibreed Population}

Covariance between relatives in a multibreed population should take account of differences in allele frequencies among breeds. The regression equation [6] should be generalized to multiple regression to take account of different expectations for mean and variance depending on breed composition of the individuals. Let $\lambda_{i k}$ denote the fraction of breed $k$ in individual $i$. Then, summing over breed combinations ( $k$ and $l$ ), equation [6] is generalized to 


$$
\mathbf{Z Z}^{\prime}=\sum_{k \leq l} b_{1(k l)} \mathbf{J}_{(k l)}+\sum_{k \leq l} b_{2(k l)} \mathbf{K}_{(k l)}+\mathbf{E},
$$

where $\mathbf{J}_{(k l)}$ and $\mathbf{K}_{(k l)}$ are breed-specific matrices. For individuals $i$ and $j$,

$$
\left(\mathbf{J}_{(k l)}\right)_{i j}= \begin{cases}\lambda_{i k} \lambda_{j k} & k=l \\ \lambda_{i k} \lambda_{j l}+\lambda_{i l} \lambda_{j k} & k<l\end{cases}
$$

are the covariates for the means (intercepts) and $\mathbf{K}_{(k l)}$ are the covariates for the regression components associated with covariances, which are determined by the rules set down in Lo et al. (1993). Namely, if $i>j$, and $s$ and $d$ denote sire and dam of $i$, then

$$
\left(\mathbf{K}_{(k l)}\right)_{i j}=0.5\left(\mathbf{K}_{(k l)}\right)_{s j}+0.5\left(\mathbf{K}_{(k l)}\right)_{d j}
$$

and

$$
\left(\mathbf{K}_{(k l)}\right)_{i i}=0.5\left(\mathbf{K}_{(k l)}\right)_{s d}+ \begin{cases}2\left(\lambda_{s k} \lambda_{s l}+\lambda_{d k} \lambda_{d l}\right) & k<l \\ \lambda_{i k} & k=l\end{cases}
$$

This algorithm is similar to that for forming the relationship matrix in a purebred population except that the diagonals are partitioned into breed fractions to account for different variances among breeds and include segregation variances because of different allele frequencies among breeds. The expectations of the regression coefficients are

$$
\begin{aligned}
& E\left(b_{1(k l)}\right)=\sum_{m}\left(p_{k m}-q_{k m}\right)\left(p_{l m}-q_{l m}\right) \\
& E\left(b_{2(k l)}\right)=\left\{\begin{array}{l}
\sum_{m}^{m} 0.5\left(p_{k m}-p_{l m}\right)^{2} \\
\sum_{m}^{m} 2 p_{k m} q_{k m}
\end{array}\right.
\end{aligned}
$$$$
k=l
$$

where $p_{k m}$ denotes allele frequency for breed $k$ at marker $m$.

The average relationship matrix may be factored as $\mathbf{A}=$ TDT $^{\prime}$ (Henderson, 1975; Quaas, 1976) and similarly

$$
\mathbf{K}_{(k l)}=\mathbf{T D}_{(k l)} \mathbf{T}^{\prime}
$$

where $\mathbf{T}$ is lower triangular containing the fractions of genes that animals derive from their ancestors and $\mathbf{D}_{(k l)}$ is diagonal containing within-family genetic variances. In addition, $\mathbf{D}=\sum_{k} \mathbf{D}_{(k k)}$ and $\mathbf{A}=\sum_{k} \mathbf{K}_{(k k)}$. The diagonal matrices can be calculated in the usual fashion

$$
\begin{aligned}
\left(\mathbf{D}_{(k l)}\right)_{i i}= & -0.25\left(\mathbf{K}_{(k l)}\right)_{s s}-0.25\left(\mathbf{K}_{(k l)}\right)_{d d} \\
& + \begin{cases}2\left(\lambda_{s k} \lambda_{s l}+\lambda_{d k} \lambda_{d l}\right) & k<l \\
\lambda_{i k} & k=l\end{cases}
\end{aligned}
$$

For example, ignoring inbreeding,

$$
\left(\mathbf{D}_{(k k)}\right)_{i i}=\lambda_{i k}- \begin{cases}0.25 \lambda_{s k}+0.25 \lambda_{d k} & \text { parents known } \\ 0.25 \lambda_{s k} & \text { dam unknown } \\ 0.25 \lambda_{d k} & \text { sire unknown } \\ 0 & \text { parents unknown }\end{cases}
$$

The pedigree used for the construction of the above relationship matrices will inevitably contain some ungenotyped ancestors. The regression coefficients in [13] were estimated using the subset of genotyped animals. Now let $\mathbf{T}_{1}$ be the submatrix of $\mathbf{T}$ comprising the subset of rows corresponding to genotyped animals; $\mathbf{T}_{1}$ is no longer lower triangular or a square matrix, but a (lower triangular) Cholesky factorization for a positivedefinite symmetric matrix can be used

$$
\mathbf{L}_{1} \mathbf{L}_{1}^{\prime}=\mathbf{T}_{1} \mathbf{D} \mathbf{T}_{1}^{\prime}=\mathbf{A}_{1}
$$

and similarly

$$
\hat{\mathbf{F}}_{1} \hat{\mathbf{F}}_{1}^{\prime}=\mathbf{T}_{1} \hat{\mathbf{D}} \mathbf{T}_{1}^{\prime}
$$

where

$$
\hat{\mathbf{D}}=\sum_{k \leq l} \hat{b}_{2(k l)} \mathbf{D}_{(k l)} .
$$

The generalization to equation [8] for estimation of the genomic relationship matrix for a multi-breed population is then given by

$$
\mathbf{G}=\mathbf{L}_{1} \hat{\mathbf{F}}_{1}^{-1}\left(\mathbf{Z Z} \mathbf{Z}^{\prime}-\sum_{k \leq l} \hat{b}_{1(k l)} \mathbf{J}_{(k l)}\right) \hat{\mathbf{F}}_{1}^{\prime-1} \mathbf{L}_{1}^{\prime} .
$$

The $\mathbf{G}$ matrix was estimated from a total of 8,706 animals in the pedigree including the 5,212 genotyped bulls. 

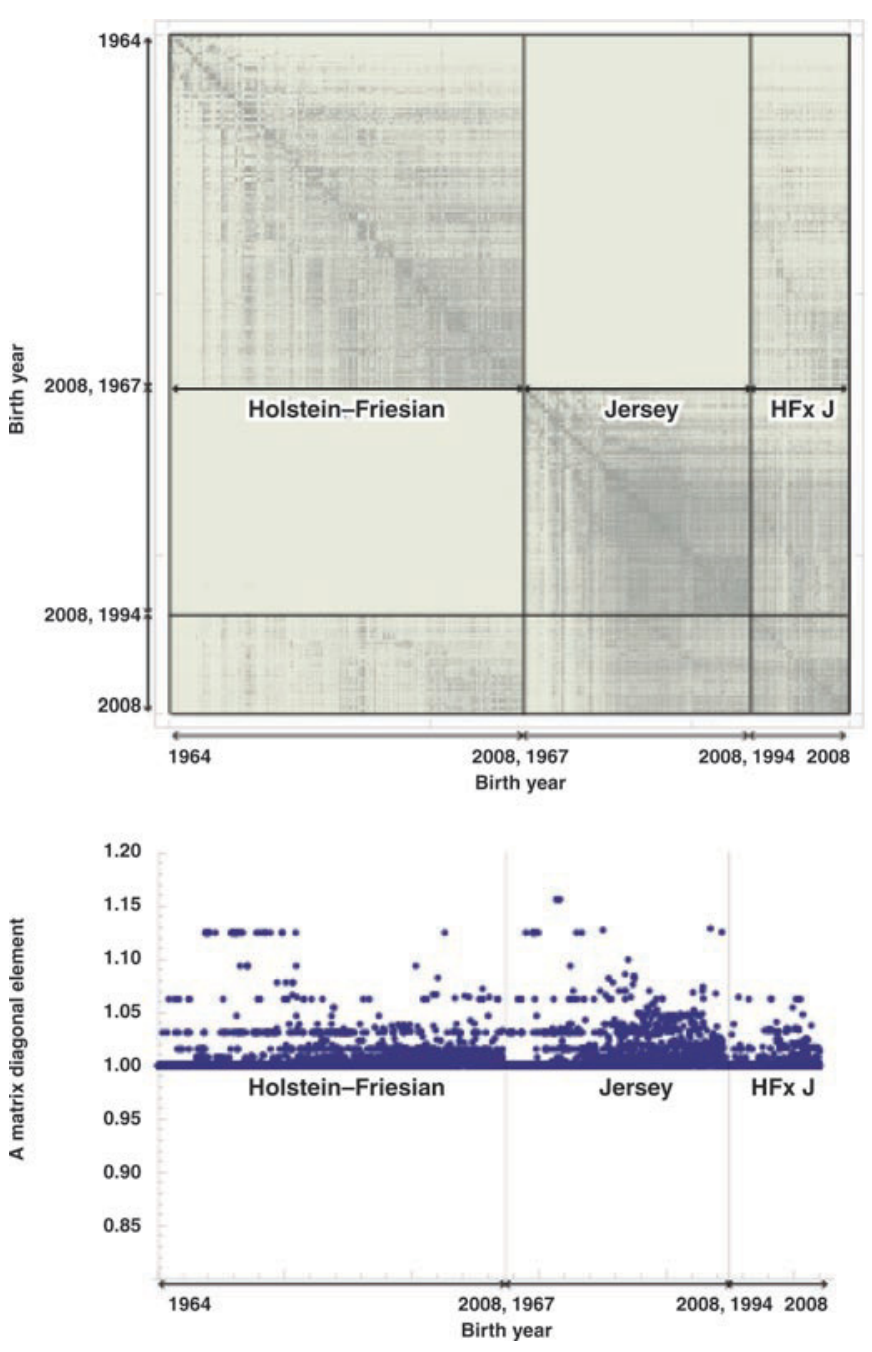

Figure 1. Heat map of genotyped block of average relationship matrix; darker areas correspond to a greater degree of relationship. The lower graph displays diagonal elements. HF = Holstein-Friesian; $\mathrm{J}=$ Jersey.

\section{Genomic Predictions}

The BV derived from the national genetic evaluation is made up of 2 components: a contribution from genetic groups (including breed effects) and a random additive genetic component, both estimated excluding SNP information. The phenotype used for the genomic predictions is the deregressed $\mathrm{BV}$ from the national genetic evaluation, first adjusted for genetic group effects,

$$
\mathbf{y}=\mathbf{R}\left(\mathbf{R}^{-1}+\sigma_{a}^{-2} \mathbf{A}^{-1}\right) \hat{\mathbf{a}}_{N},
$$

where $\hat{\mathbf{a}}_{N}$ is the random genetic component. The $\mathbf{A}$ matrix is based on the full pedigree of 8,706 animals. Equation [25] can be rewritten as

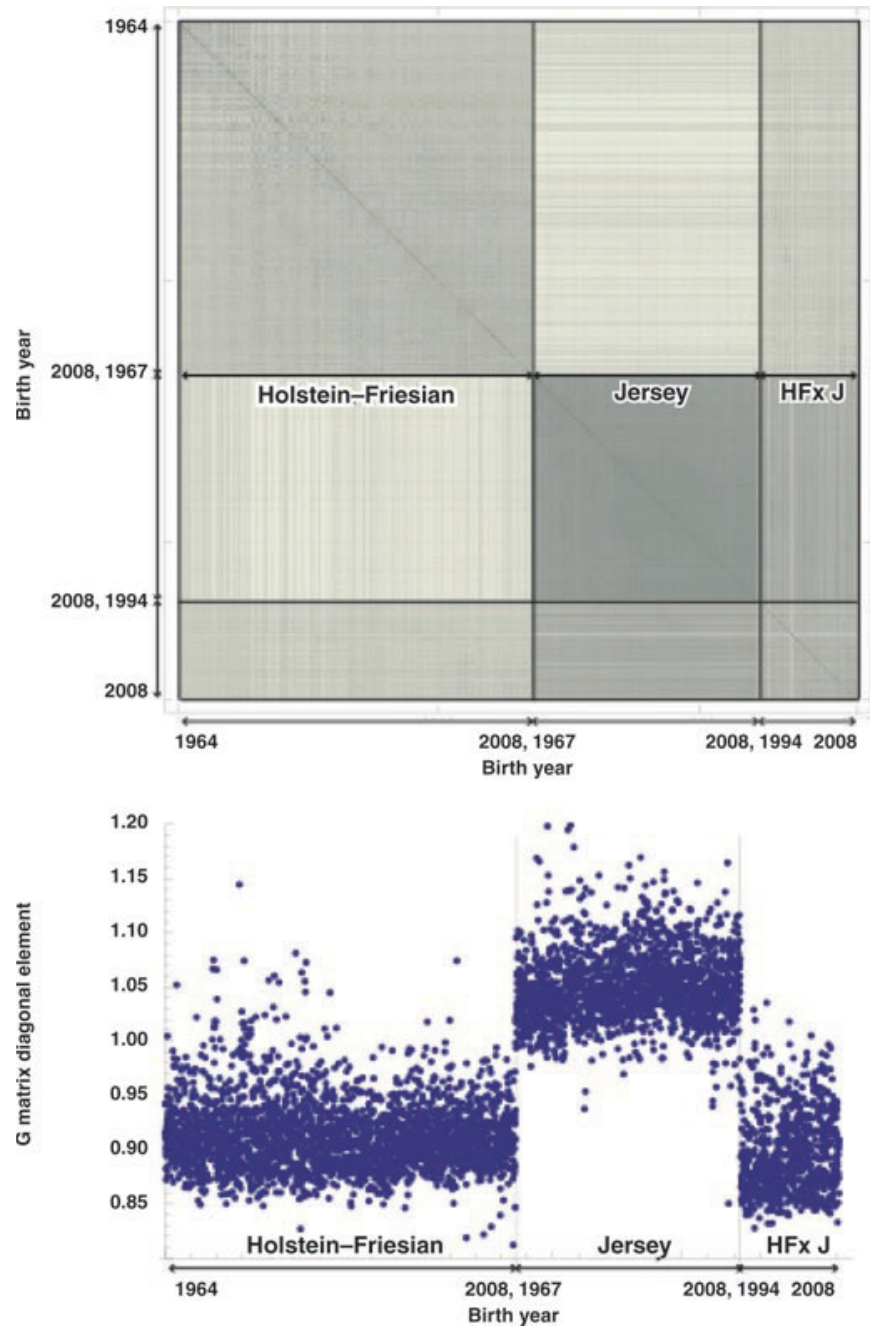

Figure 2. Heat map of genomic relationship matrix estimated ignoring breed and using whole-population SNP frequencies; darker areas correspond to a greater degree of relationship. The lower graph displays diagonal elements. HF = Holstein-Friesian; J = Jersey.

$$
\mathbf{y}=\mathbf{S}\left(\mathbf{S}^{-1}+\mathbf{A}^{-1}\right) \hat{\mathbf{a}}_{N}
$$

where

$$
\mathbf{S}^{-1}=\operatorname{diag}\left(\frac{\mathbf{r}}{1-\mathbf{r}}\right)
$$

and $\mathbf{r}$ is deregressed reliability. The method to deregress reliability is outlined in Appendix A.

Given the deregressed BV, the genomic BV (for the subset of genotyped animals) can then be estimated by the reverse process but replacing $\mathbf{A}_{1}$ by the genomic relationship matrix estimated from [24]:

$$
\hat{\mathbf{a}}_{G}=\left(\mathbf{S}_{1}^{-1}+\mathbf{G}^{-1}\right)^{-1} \mathbf{S}_{1}^{-1} \mathbf{y}_{1}=\mathbf{G}\left(\mathbf{G}+\mathbf{S}_{1}\right)^{-1} \mathbf{y}_{1},
$$



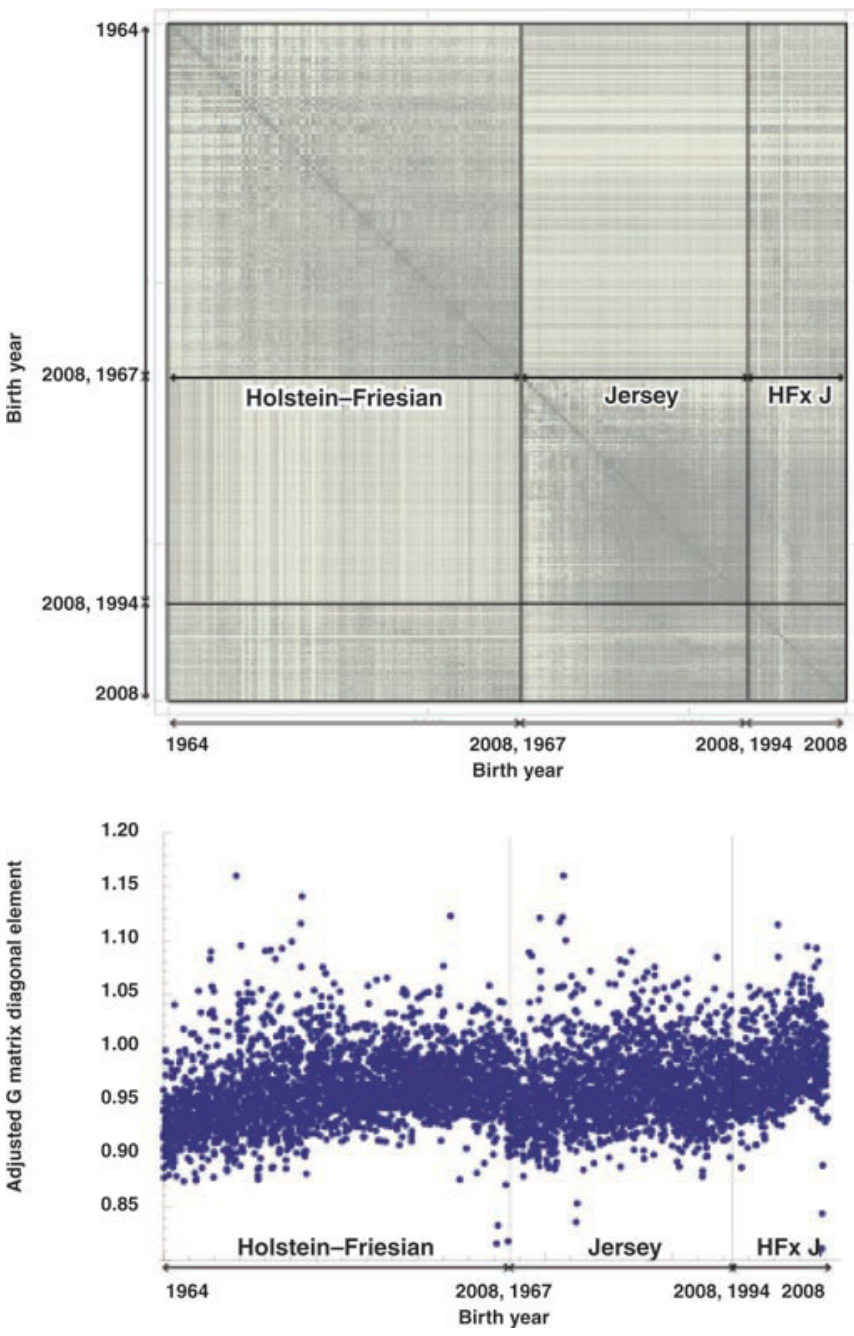

Figure 3. Heat map of genomic relationship matrix estimated using breed regression; darker areas correspond to a greater degree of relationship. The lower graph displays diagonal elements. HF = Holstein-Friesian; $\mathrm{J}=$ Jersey.

where subscript 1 refers to the subset of genotyped animals. Genomic reliabilities can be obtained from

$$
\frac{\left[\mathbf{G}\left(\mathbf{G}+\mathbf{S}_{1}\right)^{-1} \mathbf{G}\right]_{\mathrm{ii}}}{\mathbf{G}_{\mathrm{ii}}}
$$

Because the $\mathbf{G}$ matrix is based only on genotyped animals, genomic BV may not contain all the information contained in the national BV. For example, bull dam information may not be included in the "genotyped" sub-vector $\mathbf{y}_{1}$; therefore, in solving [28], bulls may lose some of the parent-average information in the national evaluation. To recapture the information lost from nongenotyped animals, predictions similar to [28] for genotyped animals based on the additive relationship matrix are carried out:

$$
\hat{\mathbf{a}}_{A}=\left(\mathbf{S}_{1}^{-1}+\mathbf{A}_{1}^{-1}\right)^{-1} \mathbf{S}_{1}^{-1} \mathbf{y}_{1}=\mathbf{A}_{1}\left(\mathbf{A}_{1}+\mathbf{S}_{1}\right)^{-1} \mathbf{y}_{1} .
$$

The 3 sources of information for genotyped animals are then combined in a selection index procedure as follows. Let $\mathbf{R}_{N}, \mathbf{R}_{A}$, and $\mathbf{R}_{G}$ be the reliabilities corresponding to $\hat{\mathbf{a}}_{N}$ (the national $\mathrm{BV}$ ), $\hat{\mathbf{a}}_{A}$ (BV based on additive relationships of genotyped), and $\hat{\mathbf{a}}_{G}$ (genomic BV), respectively. Note that $\mathbf{R}_{N} \geq \mathbf{R}_{A}$ and $\mathbf{R}_{G} \geq \mathbf{R}_{A}$. The total BV for genotyped animals is then

$$
\hat{\mathbf{g}}=\frac{\begin{array}{c}
\left(1-\mathbf{R}_{A}\right)\left(1-\mathbf{R}_{G}\right) \hat{\mathbf{a}}_{N}-\left(1-\mathbf{R}_{N}\right)\left(1-\mathbf{R}_{G}\right) \hat{\mathbf{a}}_{A} \\
+\left(1-\mathbf{R}_{N}\right)\left(1-\mathbf{R}_{A}\right) \hat{\mathbf{a}}_{G}
\end{array}}{1-2 \mathbf{R}_{A}-\mathbf{R}_{N} \mathbf{R}_{G}+\mathbf{R}_{A} \mathbf{R}_{G}+\mathbf{R}_{N} \mathbf{R}_{A}},
$$

with reliability

$$
\mathbf{R}_{g}=\frac{\mathbf{R}_{G}-\mathbf{R}_{A}+\mathbf{R}_{N}-2 \mathbf{R}_{G} \mathbf{R}_{N}+\mathbf{R}_{G} \mathbf{R}_{A} \mathbf{R}_{N}}{1-2 \mathbf{R}_{A}-\mathbf{R}_{N} \mathbf{R}_{G}+\mathbf{R}_{A} \mathbf{R}_{G}+\mathbf{R}_{N} \mathbf{R}_{A}}
$$

Details of this derivation are given in Appendix B. This gives the genomic random additive component and, finally, the genetic group effects are recombined with $\hat{\mathrm{g}}$. This is referred to as a blended BV.

\section{National Genetic Evaluation}

Consider the process for passing the genomic information through to nongenotyped individuals. Only descendants of genotyped individuals are updated in this selection index procedure. As shown in Appendix A, given the reliabilities of individual $i$ and its parents, the contribution from own records and progeny, $R_{i}^{\prime}=R_{i}(o+p)$, can be calculated and, at the same time, the parent reliabilities adjusted to exclude information from that individual, resulting in an adjusted parent average $R_{p a}^{\prime}$ reliability. Let $B V_{i}^{\prime}$ and $B V_{p a}^{\prime}$ be the corre-

Table 1. Regression estimates for genomic relationship matrix

\begin{tabular}{lcc}
\hline Breed type $^{1}$ & Intercept $\left(\hat{b}_{1}\right)$ & Slope $\left(\hat{b}_{2}\right)$ \\
\hline NZHF & 12,084 & 16,620 \\
OSHF & 12,317 & 15,095 \\
JE & 16,180 & 13,716 \\
NZHF $\times$ OSHF & 11,844 & \\
$\mathrm{NZHF} \times \mathrm{JE}$ & 10,799 & \\
OSHF $\times$ JE & 10,425 & \\
\hline
\end{tabular}

${ }^{1} \mathrm{NZHF}=$ New Zealand Holstein-Friesian; OSHF $=$ overseas Holstein; $\mathrm{JE}=$ Jersey. 
Table 2. Reliabilities (\%) of breeding values for proven bulls ${ }^{1}$

\begin{tabular}{|c|c|c|c|c|c|c|c|c|c|}
\hline Trait & \multicolumn{3}{|c|}{$\mathrm{HF}$} & \multicolumn{3}{|c|}{$\mathrm{JE}$} & \multicolumn{3}{|c|}{ FJ } \\
\hline Protein & 88.3 & 89.2 & 89.2 & 87.6 & 88.9 & 88.9 & 86.5 & 88.0 & 88.0 \\
\hline SCC & 84.0 & 85.8 & 85.8 & 81.9 & 84.7 & 84.7 & 84.3 & 86.1 & 86.1 \\
\hline Fertility & 75.3 & 78.4 & 78.4 & 73.5 & 77.9 & 77.9 & 74.3 & 78.1 & 78.1 \\
\hline
\end{tabular}

${ }^{1} \mathrm{HF}=$ Holstein-Friesian; JE $=$ Jersey; FJ $=$ Friesian $\times$ Jersey.

sponding BV. Then, the total BV can be expressed, using selection index, as

$$
\left[\begin{array}{c}
B V_{p a} \\
B V_{i}
\end{array}\right]=\frac{1}{1-R_{p a}^{\prime} R_{i}^{\prime}}\left[\begin{array}{cc}
1-0.5 R_{i}^{\prime} & 0.5-R_{p a}^{\prime} \\
1-R_{i}^{\prime} & 1-R_{p a}^{\prime}
\end{array}\right]\left[\begin{array}{c}
B V_{p a}^{\prime} \\
B V_{i}^{\prime}
\end{array}\right],
$$

where the coefficient matrix results from the matrix product of covariance matrices

$$
\left[\begin{array}{cc}
R_{p a}^{\prime} & 0.5 R_{i}^{\prime} \\
R_{p a}^{\prime} & R_{i}^{\prime}
\end{array}\right]\left[\begin{array}{cc}
R_{p a}^{\prime} & R_{i}^{\prime} R_{p a}^{\prime} \\
R_{i}^{\prime} R_{p a}^{\prime} & R_{i}^{\prime}
\end{array}\right]^{-1}
$$

Proceed down the pedigree updating the BV of ungenotyped individuals in which either parent has a BV that has been updated with genomic information. Consider an ungenotyped individual $i$ with a parent that has an updated BV. Solve [33] for $B V_{p a}^{\prime}$ and $B V_{i}^{\prime}$ given information from the national evaluation. If one or both parents has a genomic $\mathrm{BV}$, then $R_{p a}^{\prime}$ can be updated based on the incremental genomic information and an updated $B V_{p a}^{\prime}$ obtained by solving the first equation of [33] using the genomic BV for $B V_{p a}$ and keeping $B V_{i}^{\prime}$ fixed. Finally, update $B V_{i}$ using the second equation in [33] and update the reliability using the usual formula:

$$
R_{i}=\frac{R_{i}^{\prime}+R_{p a}^{\prime}-2 R_{i}^{\prime} R_{p a}^{\prime}}{1-R_{i}^{\prime} R_{p a}^{\prime}}
$$

Individual $i$ now has a genomic BV.

\section{Validation}

National BV were generated at the end of each season commencing spring 2000 through to spring 2008. Genomic (blended) BV were computed for seasons 2000 to 2007 using a genomic relationship matrix and BV appropriate to the population at each point of time. Hence, the size of the reference population is increasing with time. Proven sires received BV based on parent, progeny, and genomic information, and young sires received BV based on parents and genomics.

The top 15 unproven young bulls at 4 yr of age and within breed were "selected" based on their parentaverage BV (PABV) and also based on their genomic BV (GBV). The number of bulls within these 2 groups remaining in the top 15 based on the 2008 evaluation, including daughter information, was recorded. Also, the average protein BV of the 2 groups at the 2008 evaluation were calculated. Finally, the coefficient of determination, squared correlation between PABV or GBV at 4 yr of age, and the progeny-test BV (PTBV) at $5 \mathrm{yr}$ of age were calculated. These values were also calculated for the PTBV estimated in the 2008 evaluation, which allows more years of daughter information for older bulls.

\section{RESULTS AND DISCUSSION}

To give a relative measure of the computing times, the national genetic evaluation for protein yield using a test-day model takes approximately $24 \mathrm{~h}$ and the additional genomic evaluations approximately $15 \mathrm{~min}$. Figure 1 shows a heat map of $\mathbf{A}_{1}$, the submatrix of the

\begin{tabular}{|c|c|c|c|c|c|c|c|c|c|}
\hline \multirow[b]{2}{*}{ Trait } & \multicolumn{3}{|c|}{$\mathrm{HF}$} & \multicolumn{3}{|c|}{$\mathrm{JE}$} & \multicolumn{3}{|c|}{ FJ } \\
\hline & National & Genomic & Blended & National & Genomic & Blended & National & Genomic & Blended \\
\hline Protein & 36.2 & 54.1 & 57.9 & 35.4 & 57.0 & 60.0 & 36.2 & 56.6 & 59.4 \\
\hline Fat & 36.4 & 54.3 & 58.2 & 35.6 & 57.2 & 60.3 & 36.4 & 56.8 & 59.7 \\
\hline $\mathrm{SCC}$ & 35.3 & 52.6 & 56.4 & 34.6 & 55.2 & 58.1 & 35.4 & 54.9 & 57.7 \\
\hline Fertility & 31.7 & 48.1 & 51.1 & 31.6 & 50.7 & 53.1 & 32.2 & 50.5 & 52.6 \\
\hline
\end{tabular}
average relationship matrix corresponding to the 5,212

Table 3. Reliabilities (\%) of breeding values for unproven bulls ${ }^{1}$

${ }^{1} \mathrm{HF}=$ Holstein-Friesian; JE $=$ Jersey; FJ $=$ Friesian $\times$ Jersey. 
Table 4. Reliabilities of blended breeding values for nonproduction traits ${ }^{1}$

\begin{tabular}{|c|c|c|c|c|c|c|}
\hline \multirow[b]{2}{*}{ Trait } & \multicolumn{3}{|c|}{ Proven bulls } & \multicolumn{3}{|c|}{ Unproven bulls } \\
\hline & $\mathrm{HF}$ & JE & FJ & $\mathrm{HF}$ & $\mathrm{JE}$ & FJ \\
\hline Adaptability & 85.2 & 84.8 & 85.1 & 54.1 & 56.9 & 55.5 \\
\hline Temperament & 85.1 & 84.7 & 84.9 & 54.0 & 56.8 & 55.4 \\
\hline Milking speed & 81.1 & 80.9 & 80.6 & 51.3 & 54.1 & 52.8 \\
\hline Overall opinion & 85.3 & 84.9 & 85.1 & 54.1 & 57.0 & 55.5 \\
\hline Stature & 88.7 & 88.1 & 89.0 & 56.8 & 59.4 & 57.9 \\
\hline Capacity & 86.3 & 85.7 & 86.4 & 55.1 & 57.7 & 56.2 \\
\hline Rump angle & 85.4 & 84.7 & 85.3 & 54.4 & 57.0 & 55.6 \\
\hline Rump width & 85.2 & 84.6 & 85.2 & 54.3 & 56.9 & 55.5 \\
\hline Legs & 74.1 & 73.9 & 73.1 & 47.0 & 49.5 & 48.3 \\
\hline Udder support & 89.2 & 88.5 & 89.5 & 57.1 & 59.8 & 58.2 \\
\hline Fore udder & 87.8 & 87.1 & 88.0 & 56.1 & 58.7 & 57.3 \\
\hline Rear udder & 87.7 & 87.0 & 87.9 & 56.0 & 58.6 & 57.2 \\
\hline Front teat & 88.0 & 87.3 & 88.2 & 56.2 & 58.9 & 57.4 \\
\hline Rear teat & 87.9 & 87.3 & 88.2 & 56.2 & 58.8 & 57.3 \\
\hline Udder overall & 83.6 & 83.0 & 83.4 & 53.2 & 55.7 & 54.4 \\
\hline Dairy conformation & 87.1 & 86.4 & 87.2 & 55.6 & 58.2 & 56.7 \\
\hline
\end{tabular}

${ }^{1} \mathrm{HF}=$ Holstein-Friesian; JE $=$ Jersey; FJ $=$ Friesian $\times$ Jersey.

genotyped bulls. Darker areas correspond to a greater degree of relationship, which is evident in the JE subpopulation relative to HF. Figure 2 shows the genomic relationship matrix estimated using equation [8], where the regression estimates were replaced by their expected values [equation 7] and the allele frequencies were estimated across the whole population. The values were 12,276 and 14,983 for intercept and slope, respectively, corresponding to 42,302 SNP. Figure 2 differs markedly from Figure 1; the $\mathbf{G}$ matrix estimated ignoring breed is not in the neighborhood of its expected value, the $\mathbf{A}$ matrix. The diagonal elements shown at the bottom of each figure illustrate the distortion apparent in Figure 2 from using common parameters across breed, with the majority of HF values being less than unity and vice versa for JE. Finally, Figure 3, based on equation [24], has greater similarity to Figure 1. Three breed groupings were used: overseas HF, New Zealand HF, and JE. The regression estimates based on equation [13] are given in Table 1 and have expectation based on [17]. When divided by the number of SNP $(42,302)$, the values in Table 1 reflect average values of the summands in [17]. The terms corresponding to segregation variances in [13] were not included in the final model because of difficulty with small negative estimates of the regression coefficients; the power of estimation is low because of few backcross or $\mathrm{F}_{2}$ animals. The corresponding entries are therefore blank in Table 1. These results confirm the need to take account of breed for the estimation of the genomic relationship matrix in a crossbred population. An attempt to estimate regression parameters from equation [6] ignoring breed resulted in a negative estimate of the slope parameter.

Consider a young bull with national parent-average reliability $R_{N}=0.3$, "reduced information" parent-average $R_{A}=0.25$, and genomic $\mathrm{BV} R_{G}=0.55$, then using equations [31] and [32], $\hat{g}=0.62 \hat{a}_{N}-0.57 \hat{a}_{A}+0.96 \hat{a}_{G}$ with reliability $R_{g}=0.57$. The influence of the parentaverage adjustment in the final result is $1-R_{G} / R_{q}$, which is $3.3 \%$. Tables 2 and 3 give average predicted reliabilities for some traits in the New Zealand national Breeding Worth economic index. In general, for proven bulls, reliabilities increased 1 to $4 \%$ with genomic information. For unproven bulls, the national evaluation parent average of $35 \%$ reliability increased on average

Table 5. Number of young bulls first selected on parent-average breeding value (PABV) or genomic breeding value (GBV) remaining in the top 15 after progeny test for protein yield (2008 season)

\begin{tabular}{|c|c|c|c|c|c|c|}
\hline \multirow[b]{2}{*}{ Breed $^{1}$} & \multicolumn{2}{|c|}{2000 born } & \multicolumn{2}{|c|}{2002 born } & \multicolumn{2}{|c|}{2004 born } \\
\hline & PABV & GBV & PABV & GBV & PABV & GBV \\
\hline $\mathrm{HF}$ & 4 & 9 & 6 & 10 & 3 & 7 \\
\hline JE & 7 & 8 & 5 & 9 & 9 & 10 \\
\hline FJ & 9 & 9 & 7 & 9 & 8 & 8 \\
\hline
\end{tabular}

${ }^{1} \mathrm{HF}=$ Holstein-Friesian; JE $=$ Jersey; FJ $=$ Friesian $\times$ Jersey. 
Table 6. Average protein BV (2008 season) of 15 young bulls first selected on parent-average breeding value (PABV) or genomic breeding value (GBV)

\begin{tabular}{|c|c|c|c|c|c|c|}
\hline \multirow[b]{2}{*}{ Breed $^{1}$} & \multicolumn{2}{|c|}{2000 born } & \multicolumn{2}{|c|}{2002 born } & \multicolumn{2}{|c|}{2004 born } \\
\hline & PABV & GBV & PABV & GBV & PABV & GBV \\
\hline $\mathrm{HF}$ & 28.8 & 31.6 & 28.9 & 33.5 & 33.9 & 37.3 \\
\hline JE & 0 & 1.0 & 0.5 & 2.4 & 7.5 & 9.4 \\
\hline FJ & 12.7 & 14.2 & 13.1 & 15.7 & 21.9 & 21.4 \\
\hline
\end{tabular}

${ }^{1} \mathrm{HF}=$ Holstein-Friesian; JE $=$ Jersey; FJ $=$ Friesian $\times$ Jersey.

Table 7. Coefficient of determination $(\times 100)$ between parent-average breeding value $(\mathrm{PABV})$ or genomic breeding value (GBV) as $4 \mathrm{yr}$ old and progeny-test breeding values as $5 \mathrm{yr}$ old for protein yield

\begin{tabular}{lcccccccc}
\hline & \multicolumn{2}{c}{2000 born } & & \multicolumn{2}{c}{2002 born } & & \multicolumn{2}{c}{2004 born } \\
\cline { 2 - 3 } Breed $^{1}$ & PABV & GBV & & PABV & GBV & & PABV & GBV \\
\hline HF & 42 & 56 & & 25 & 45 & & 31 & 46 \\
JE & 28 & 46 & & 23 & 41 & & 34 & 48 \\
FJ & 51 & 66 & & 42 & 62 & & 32 & 48 \\
\hline
\end{tabular}

${ }^{1} \mathrm{HF}=$ Holstein-Friesian; JE $=$ Jersey; FJ $=$ Friesian $\times$ Jersey.

to $54 \%$ with genomic information and further to $57 \%$ with blending. The figures are lower for fertility. Table 4 gives average reliabilities of blended BV for nonproduction traits.

Table 5 shows that of the top 15 young bulls selected on parent average, an average of 6.4 were in the top 15 after daughter information was included. For selection on GBV, this figure increased to 8.7. From Table 6, the average increase in protein BV (2008 evaluation) due to selecting the team on GBV compared with PABV was $2.1 \mathrm{~kg}$. Genomic predictions increased coefficients of determination for production traits. Coefficients of determination were 14 to $20 \%$ higher for GBV relative to PABV when comparing to first PTBV (Table 7 ) and 4 to $26 \%$ higher when comparing to the PTBV at the 2008 evaluation (Table 8). If the figures in Table 7 are divided by a typical range of 0.8 to 0.9 for the reliability of PTBV at $5 \mathrm{yr}$ of age (using a higher value for older bulls), then a realized reliability is estimated for the GBV (Hayes et al., 2009), which correspond to the predicted reliabilities for fat and protein included in Table 3.
The availability of genome-wide dense marker maps for dairy cattle has allowed GBV to be estimated on young sires with no progeny information and, based on the increased accuracy of GBV indicated above, has facilitated the marketing of teams of bulls including some as young as 2 yr of age. It may be possible to increase the accuracy of GBV. Factors affecting accuracy include the level of linkage disequilibrium between markers and QTL, and the number of animals with both phenotypes and genotypes. Denser SNP chips should result in increased linkage disequilibrium between markers and QTL, thereby increasing the proportion of genetic variance explained by the markers. As more phenotypic records are collected, the size of the reference population will increase, providing more observations for the estimation of each SNP allele. Genotyping of some dams of young bulls is being carried out, which will reduce the need for the blending step. Other opportunities for increased accuracy may include relaxation of the assumption of equal variance across all SNP and the feature selection of a subset of SNP that explain most of the genetic variation. The national genetic evalua-

Table 8. Coefficient of determination $(\times 100)$ between parent-average breeding value $(\mathrm{PABV})$ or genomic breeding value (GBV) as $4 \mathrm{yr}$ old and progeny-test breeding value at 2008 evaluation for protein yield

\begin{tabular}{lcccccccc}
\hline & \multicolumn{2}{c}{2000 born } & & \multicolumn{2}{c}{2002 born } & & \multicolumn{2}{c}{2004 born } \\
\cline { 2 - 3 } Breed $^{1}$ & PABV & GBV & & PABV & GBV & & PABV & GBV \\
\hline HF & 34 & 44 & & 18 & 31 & & 17 & 26 \\
JE & 19 & 31 & & 14 & 22 & & 31 & 35 \\
FJ & 42 & 62 & & 27 & 53 & & 20 & 33 \\
\hline
\end{tabular}

${ }^{1} \mathrm{HF}=$ Holstein-Friesian; JE $=$ Jersey; FJ $=$ Friesian $\times$ Jersey. 
tion currently includes an effect for heterosis. Improved performance because of nonadditive effects is based on average heterotic effects. Genomic data may provide an opportunity for the separation of the additive and nonadditive components allowing the optimization of specific crosses, which has not been feasible under classical breeding programs.

This paper has outlined a method for estimation of genomic BV in a multibreed population combining mixed model methodology with selection index and then permeating this information to descendants in the commercial population. A future challenge will be solving large-scale national genetic evaluations that include genomic information in one step. The size of the genomic relationship matrix, which is a dense matrix, will increase with time and provide challenges for the future.

\section{REFERENCES}

Habier, D., R. L. Fernando, and J. C. M. Dekkers. 2007. The impact of genetic relationship information on genome-assisted breeding values. Genetics 177:2389-2397.

Harris, B. L., and D. L. Johnson. 1998. Approximate reliability of genetic evaluations under an animal model. J. Dairy Sci. 81:27232728 .

Hayes, B. J., P. J. Bowman, A. J. Chamberlain, and M. E. Goddard. 2009. Invited review: Genomic selection in dairy cattle: Progress and challenges. J. Dairy Sci. 92:433-443.

Henderson, C. R. 1975. Rapid method for computing the inverse of a relationship matrix. J. Dairy Sci. 58:1-8.

Lo, L. L., R. L. Fernando, and M. Grossman. 1993. Covariance between relatives in multibreed populations: Additive model. Theor. Appl. Genet. 87:423-430.

Meuwissen, T. H. E. 2007. Genomic selection: Marker assisted selection on a genome wide scale. J. Anim. Breed. Genet. 124:321-322.

Meuwissen, T. H. E., B. H. Hayes, and M. E. Goddard. 2001. Prediction of total genetic value using genome-wide dense marker maps. Genetics 157:1819-1829.

Quaas, R. L. 1976. Computing the diagonal elements and inverse of a large numerator relationship matrix. Biometrics 32:949-953.

Schaeffer, L. R. 2006. Strategy for applying genome-wide selection in dairy cattle. J. Anim. Breed. Genet. 123:218-223.

Van Tassell, C. P., T. P. L. Smith, L. K. Matukumalli, J. F. Taylor, R. D. Schnabel, C. Taylor Lawley, C. D. Haudenschild, S. S. Moore, W. C. Warren, and T. S. Sonstegard. 2008. SNP discovery and allele frequency estimation by deep sequencing of reduced representation libraries. Nat. Methods 5:247-252.

VanRaden, P. M. 2008. Efficient methods to compute genomic predictions. J. Dairy Sci. 91:4414-4423.

VanRaden, P. M., C. P. Van Tassel, G. R. Wiggans, T. S. Sonstegard, R. D. Schnabel, J. F. Taylor, and F. S. Schenkel. 2009. Invited review: Reliability of genomic predictions for North American Holstein bulls. J. Dairy Sci. 92:16-24.

\section{APPENDIX A}

\section{Deregression of Reliability}

The method is the reverse of the process for building reliability as presented in Harris and Johnson (1998).
First, remove the influence of parents from an individual's reliability. The contribution of the parent average to an individual's reliability is given by (Harris and Johnson, 1998)

$$
R_{p a}^{\prime}=0.25\left(R_{s}^{\prime}+R_{d}^{\prime}\right)
$$

where $R_{s}^{\prime}$ is the contribution from the sire (excluding the individual) and is given by

$$
R_{s}^{\prime}=\frac{R_{s}-0.25 R_{i}(o+p)}{1-0.5 R_{i}(o+p)+0.25 R_{s} R_{i}(o+p)},
$$

and similarly for the dam where $R_{s}$ is the sire's total reliability and $R_{i}(o+p)$ is the individual's reliability based on own records and progeny. The individual's total reliability is given by

$$
R_{i}=\frac{R_{i}(o+p)+R_{p a}^{\prime}-2 R_{i}(o+p) R_{p a}^{\prime}}{1-R_{i}(o+p) R_{p a}^{\prime}} .
$$

Set $x=R_{i}(o+p)$ and rearrange the last equation to get

$$
x=R_{i}-R_{p a}^{\prime}\left(1-2 x+x R_{i}\right)
$$

where

$$
R_{s}^{\prime}=\frac{R_{s}-0.25 x}{1-0.5 x+0.25 x R_{s}}
$$

and similarly for $R_{d}^{\prime}$. Thus, given $R_{i}, R_{s}$, and $R_{d}$, one can solve for $x$ by repeated substitution until convergence initially setting $x=R_{i}-0.25\left(R_{s}+R_{d}\right)$. This process proceeds down the pedigree from oldest to youngest.

The second stage involves removing information based on progeny. Starting from the youngest generation, the reliability based on own records $R_{i}(o)$ is

$$
R_{i}(o)=\frac{R_{i}(o+p)-R_{i}(p)}{1-2 R_{i}(p)+R_{i}(o+p) R_{i}(p)},
$$

where $R_{i}(p)$ is reliability based on progeny only. $R_{i}(p)$ is built up at the same time as $R_{i}(o)$ becomes available for the younger generations. 


\section{APPENDIX B}

\section{Selection Index to Combine Genomic Information with Other Pedigree and Performance Information}

The marginal reliability of the information in $\hat{a}_{G}$ given $\hat{a}_{A}$ (Harris and Johnson, 1998) is

$$
R_{G \mid A}=\frac{R_{G}-R_{A}}{1+R_{G} R_{A}-2 R_{A}} .
$$

Let $\hat{a}_{G} \mid \hat{a}_{A}$ represent the additional BV information in $\hat{a}_{G}$ given $\hat{a}_{A}$. Using selection index

$$
\begin{aligned}
\hat{a}_{G} & =\left[\begin{array}{ll}
\hat{a}_{A} & \hat{a}_{G} \mid \hat{a}_{A}
\end{array}\right]\left[\begin{array}{cc}
R_{A} & R_{A} R_{G \mid A} \\
R_{A} R_{G \mid A} & R_{G \mid A}
\end{array}\right]^{-1}\left[\begin{array}{c}
R_{A} \\
R_{G \mid A}
\end{array}\right] \\
& =\frac{\left(1-R_{G \mid A}\right) \hat{a}_{A}+\left(1-R_{A}\right) \hat{a}_{G} \mid \hat{a}_{A}}{1-R_{A} R_{G \mid A}} \\
& =\frac{1-R_{G}}{1-R_{A}} \hat{a}_{A}+\frac{1+R_{G} R_{A}-2 R_{A}}{1-R_{A}} \hat{a}_{G} \mid \hat{a}_{A},
\end{aligned}
$$

and similarly

$$
\hat{a}_{N}=\frac{1-R_{N}}{1-R_{A}} \hat{a}_{A}+\frac{1+R_{N} R_{A}-2 R_{A}}{1-R_{A}} \hat{a}_{N} \mid \hat{a}_{A} .
$$

Now, use the above to get the covariance between the genomic BV and national BV:

$$
\operatorname{cov}\left(\hat{a}_{G}, \hat{a}_{N}\right)=R_{G} R_{N}+\frac{\left(1-R_{G}\right)\left(1-R_{N}\right) R_{A}}{1-R_{A}} .
$$

Note that this can also be expressed as

$$
\operatorname{cov}\left(\hat{a}_{G}, \hat{a}_{N}\right)=R_{A}+\frac{\left(R_{G}-R_{A}\right)\left(R_{N}-R_{A}\right)}{1-R_{A}},
$$

which is equivalent to the corresponding covariance in VanRaden et al. (2009).

Combining the 3 bits of information $\hat{a}_{N}, \hat{a}_{A}$, and $\hat{a}_{G}$ again using selection index

$$
\begin{aligned}
\hat{g} & =\left[\begin{array}{lll}
\hat{a}_{N} & \hat{a}_{A} & \hat{a}_{G}
\end{array}\right]\left[\begin{array}{ccc}
R_{N} & R_{A} & R_{A}+\frac{\left(R_{G}-R_{A}\right)\left(R_{N}-R_{A}\right)}{1-R_{A}} \\
R_{A} & R_{A} & R_{A} \\
R_{A}+\frac{\left(R_{G}-R_{A}\right)\left(R_{N}-R_{A}\right)}{1-R_{A}} & R_{A} & R_{G}
\end{array}\right]^{-1} \\
= & \frac{\left(1-R_{A}\right)\left(1-R_{G}\right) \hat{a}_{N}-\left(1-R_{N}\right)\left(1-R_{G}\right) \hat{a}_{A}+\left(1-R_{N}\right)\left(1-R_{A}\right) \hat{a}_{G}}{1-2 R_{A}-R_{N} R_{G}+R_{A} R_{G}+R_{N} R_{A}}
\end{aligned}
$$

with reliability

$$
\frac{\left[\begin{array}{lll}
R_{N} & R_{A} & R_{G}
\end{array}\right]\left[\begin{array}{c}
\left(1-R_{A}\right)\left(1-R_{G}\right) \\
-\left(1-R_{N}\right)\left(1-R_{G}\right) \\
\left(1-R_{N}\right)\left(1-R_{A}\right)
\end{array}\right]}{1-2 R_{A}-R_{N} R_{G}+R_{A} R_{G}+R_{N} R_{A}}
$$

